\title{
Research of filtration and energy parameters of membrane bioreactors
}

\author{
Nikolay Makisha* \\ Moscow State University of Civil Engineering, 26, Yaroslavskoye shosse, Moscow, Russia, 129337
}

\begin{abstract}
Membrane bioreactors nowadays are intensively applied in wastewater treatment worldwide. The paper refers to a research, which goal was to evaluate the optimal parameters of membrane bioreactor performance. A focus was given to a part of the research that considered operation of side-stream membrane bioreactor under pressure of $0.2 \mathrm{MPa}$ and variable amount of backwashes. There were three types of feed water investigated with mixed liquor suspended solids concentrations of 1.6, 2.5 and $7 \mathrm{~g} / \mathrm{L}$, respectively. The article provides results of permeate production and their processing to obtain specific energy consumption for each combination of operation parameters.
\end{abstract}

\section{Introduction}

Application of membrane bioreactors for wastewater treatment, which became well known in 1980 s, is now a matter of a significant interest from viewpoint of research and practical implementation [1,2]. The more detailed the research are, the wider and more solid data will be obtained and the more accurate will be the operation. Real performance, of course, cannot be fully predicted during the bench-scale, pilot-scale or even full-scale research, however, all of these can significantly contribute to sustainable operation $[3,4]$. The experience in operation of MBR in Russian Federation is not so solid, and these blanks and gaps should be filled [5]. It considers almost every aspect of MBR - contamination removal from wastewater (with typical for Russia values of pollutants), energy efficiency of the process, fouling mitigation, operation conditions and many others [6,7]

If membrane bioreactors are taken into account submersible MBR are the most common solution in majority of cases. On the other hand, if to apply MBRs for reconstruction of small wastewater treatment plants (WWTP) the facilities' dimensions always matter and dimensions of auxiliary tanks in particular $[8,9]$. In this viewpoint side-stream membrane bioreactors can be focused on within a research as a probable option if to place them nearby in portable containers (and without auxiliary capital construction) [10].

\footnotetext{
* corresponding author - nmakisha@gmail.com
} 


\section{Materials and methods}

The research was performed following a goal to investigate operation of side-stream MBR in case of several possible operation parameters - mixed liquor suspended solids (MLSS), operation pressure, and amount of backwashes. The objectives of the research are:

- $\quad$ to study suspended particles detention (of active sludge) using membrane methods at different values of operating pressure

- to increase performance of membrane bioreactors, as well as to determine the optimal range of working pressure under which it is possible to prevent clogging of membranes and breakthrough of the contaminants in the filtrate.

The bench scheme is shown in figure 1. The feed wastewater (mixed liquor after biological treatment) is supplied from the feed water tank 1 to the membrane module 3 using the pump 2. The working pressure is regulated by means of a pressure tank 4 , pressure switch 5, a bypass valve 10, and a pressure gauge 12. Permeate after the membrane module was collected in the samplers 7. Ordinary capillary ultrafiltration membranes with standard characteristics were used for the bench-scale research with pore size of 0.1 micron and total surface area of $1 \mathrm{~m}^{2}$.

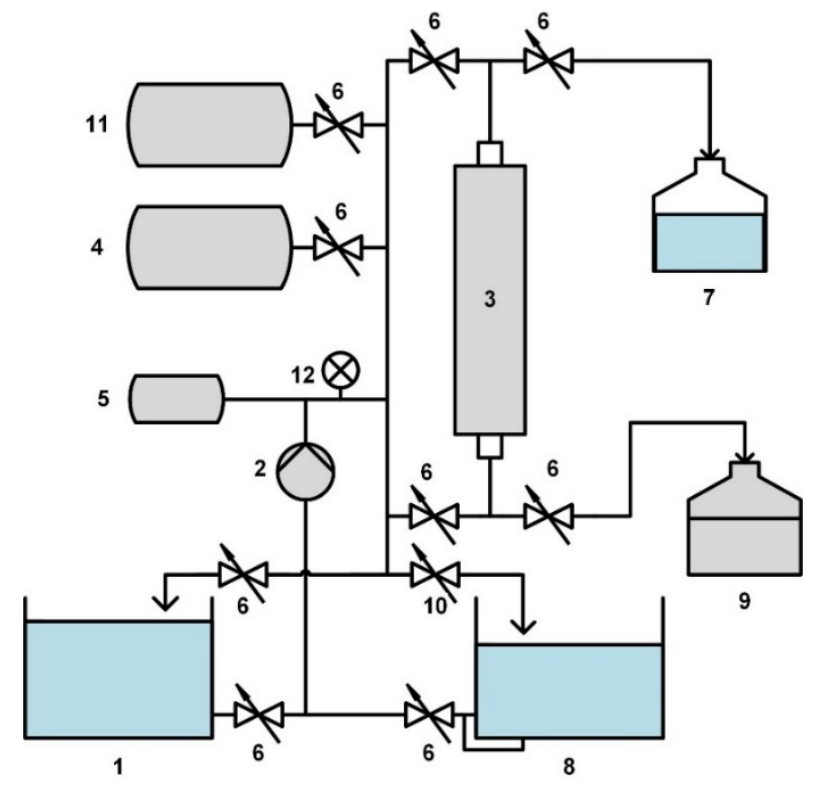

Fig. 1. Scheme of the bench-scale model: 1 - feed water tank; 2 - pump; 3 - membrane module; 4 pressure tank; 5 - pressure switch; 6 - valve; 7 - sampler tank; 8 - washing tank; 9 -washing water sampler; 10 - bypass valve; 11 - pressure tank for washing; 12 - pressure gauge

Backwash is a mandatory option for the functioning of membrane modules in MBR. Deionized water after the reverse osmosis plant was used for backwashing and supplied from washing tank 8 . Switching between feed and wash tanks was performed using valves 6. The working pump fed the wash water into the filtrate path; afterwards it was collected through the source water path to the wash water samplers 9. Switching of pipelines was carried out by means of valves 6 . Pressure was controlled with pressure switch 5 , a bypass valve 10 and pressure tank of washing water 11 .

The whole plot of the research was divided into several sub-stages to compare obtained results in the following. The criteria of the division was operation pressure, while the MLSS value remained the same through the parts of the research. There were three 
concentrations of MLSS investigated within the research: MLSS=1.6 and $2.5 \mathrm{~g} / \mathrm{L}$ are similar to those in conventional activated sludge systems. This approach seems reasonable, if the MBR will be applied for reconstruction of existing WWTP with current operation parameters. The third value, MLSS $=7 \mathrm{~g} / \mathrm{L}$ lies in a range of optimal values for submerged MBR. This paper has a focus on a part of the research with operation pressure of $0.2 \mathrm{MPa}$, as our previous study showed that higher operation pressure stimulates breakthrough of suspended solids [11].

\section{Results}

The first obtained results were the volumes of permeate related to variable duration of membrane filtration (Figure 2). It is predictable, that higher efficiency (greater permeate volume) was achieved at low MLSS value (1.6 g/L). Slightly higher MLSS (of $2.5 \mathrm{~g} / \mathrm{L}$ ) provided a slight (10-15\%) decrease of permeate production, however, both sets of values may be considered as comparable. At the meantime, the permeate production dropped 3.2 times when the MLSS value was risen up to $7 \mathrm{~g} / \mathrm{L}$ comparing to MLSS=1.6 g/L.

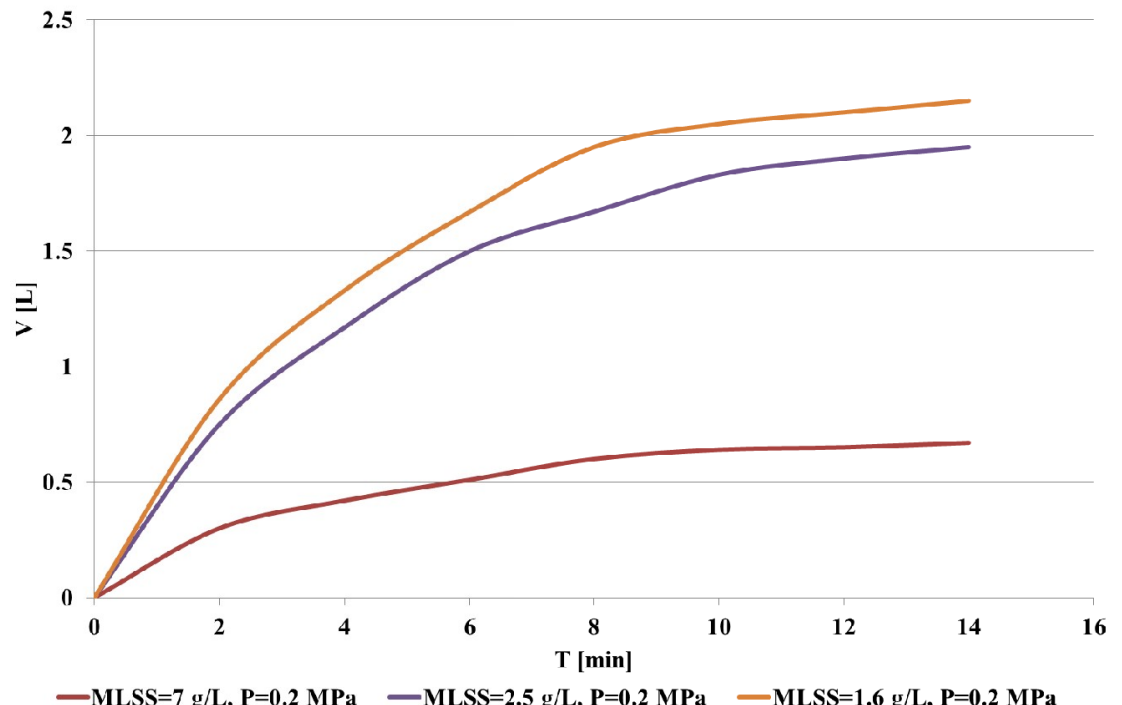

Fig. 2. Growth of permeate volume through time

That may signal that under operation pressure of $0.2 \mathrm{MPa}$ the permeate production is sharply depended on the growth of MLSS. In other words, the permeate production plummets due to soar of fouling resistance cause by intensive pore clogging. The most intensive growth of permeate production is witnessed at the initial period of filtration almost linear growth can be seen within first 3 minutes of filtration (for all three MLSS). The next part is filtration between 3 and 8 minutes, when the permeate production grows by approximately $70 \%$ under all three MLSS value. Duration of 8 minutes is a sort of checkpoint as far as the next 5 minutes period showed only $10 \%$ of permeate production growth. That means that after 8 minutes of filtration the cake layer on the membrane surface is dense enough to stop the filtration at any MLSS value. The same process can also be described through the dependence between flow and time of filtration (figure 3). The higher the MLSS value the more intensive is flux drop due to rapid pore fouling. Permeate volume in certain time can be determined if to draw straight line from abscissa axis and 
parallel to ordinate axis. Area of obtained figure corresponds to volume of filtered wastewater during selected time.

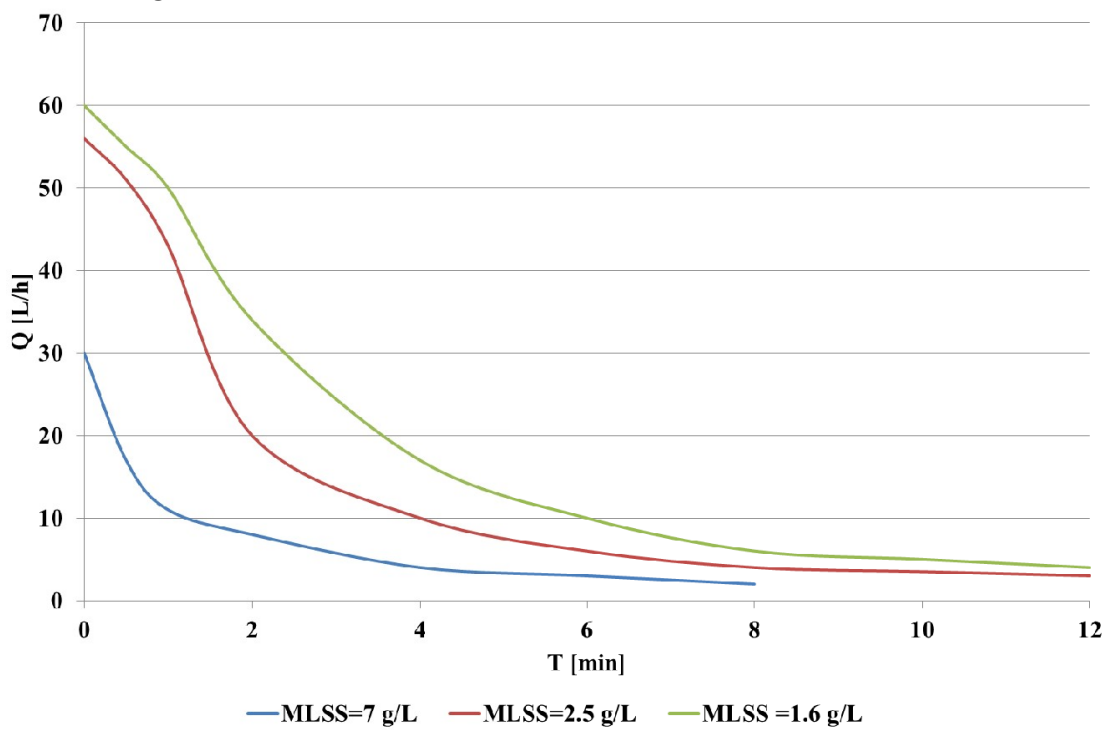

Fig. 3. Flux decrease within filtration cycle (pressure $=0.2 \mathrm{MPa}$ )

For our further investigation of operation conditions, we focused at three possible operation modes - filtration cycle duration of 2, 4 and 6 minutes or 30,15 and 10 backwashes respectively (figure 4).

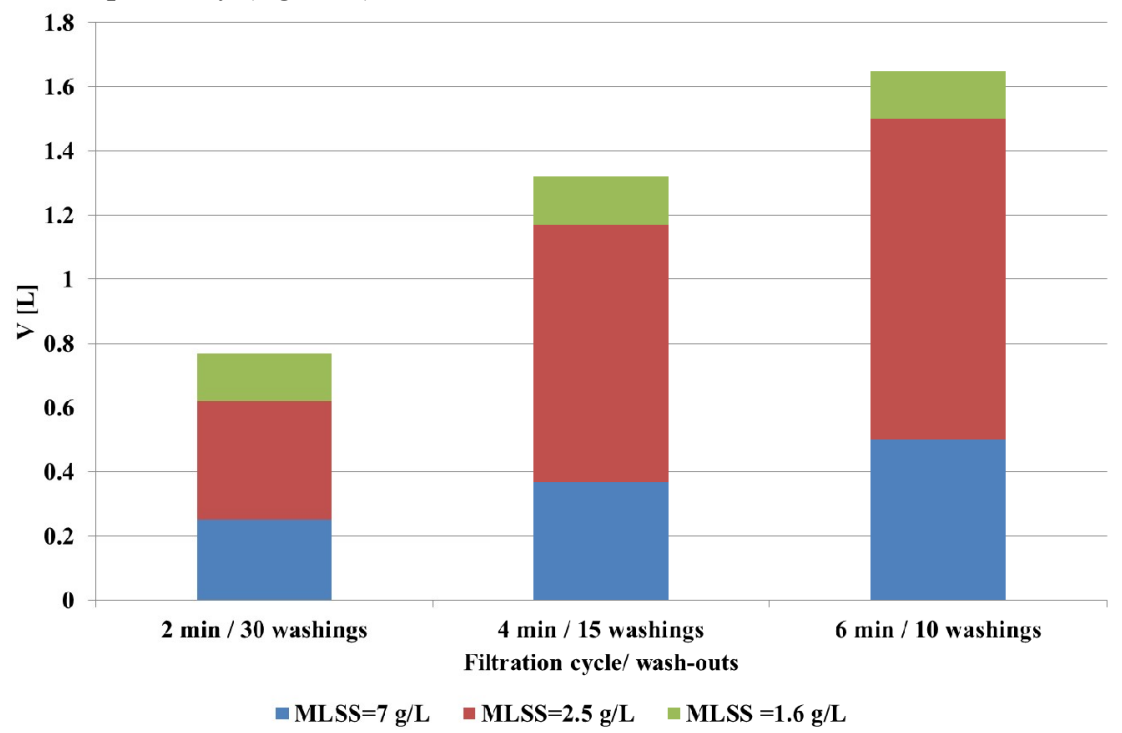

Fig. 4. Permeate volume different operation conditions

It is obvious that the shorter filtration cycle is the larger is permeating volume. On the other hand, the more often backwashes are applied, the larger is the volume of water required to implement efficient backwash and the lower is "useful" product water amount defined as a difference between total permeate amount and water used for backwashing. 
Backwash efficiency depends on filtration pressure value and time period of backwash. In addition, backwash efficiency can be evaluated as percentage of fouling (suspended solids) removed from membrane surface. Definition of balanced duration of filtration that corresponds to minimal operational costs was among the goals of our research.

Experimental data processing for experiments are presented on figures 5 and 6 . Figure 5 shows dependencies of specific membrane flow $\left(\mathrm{Lh}^{-1}\right)$ to MLSS concentration and frequency of backwashes. Total permeate flow of membrane module decreases with the increase of suspended solids (and fouling, respectively) concentration.

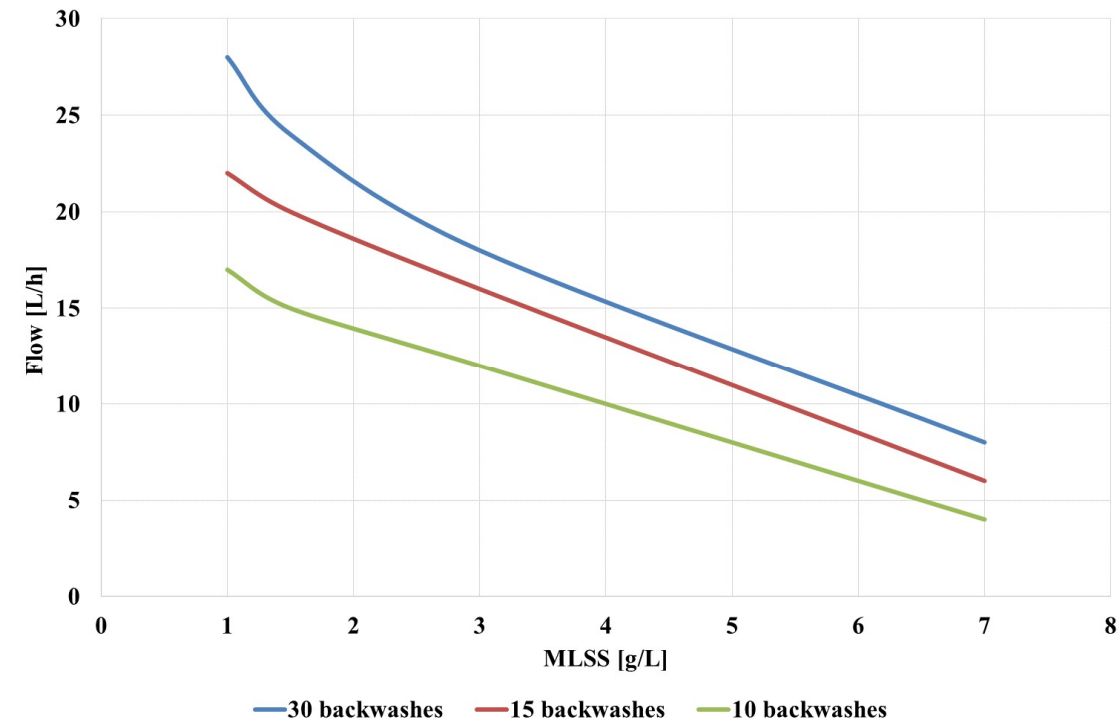

Fig.5. Flux determination in case of different backwashes amount

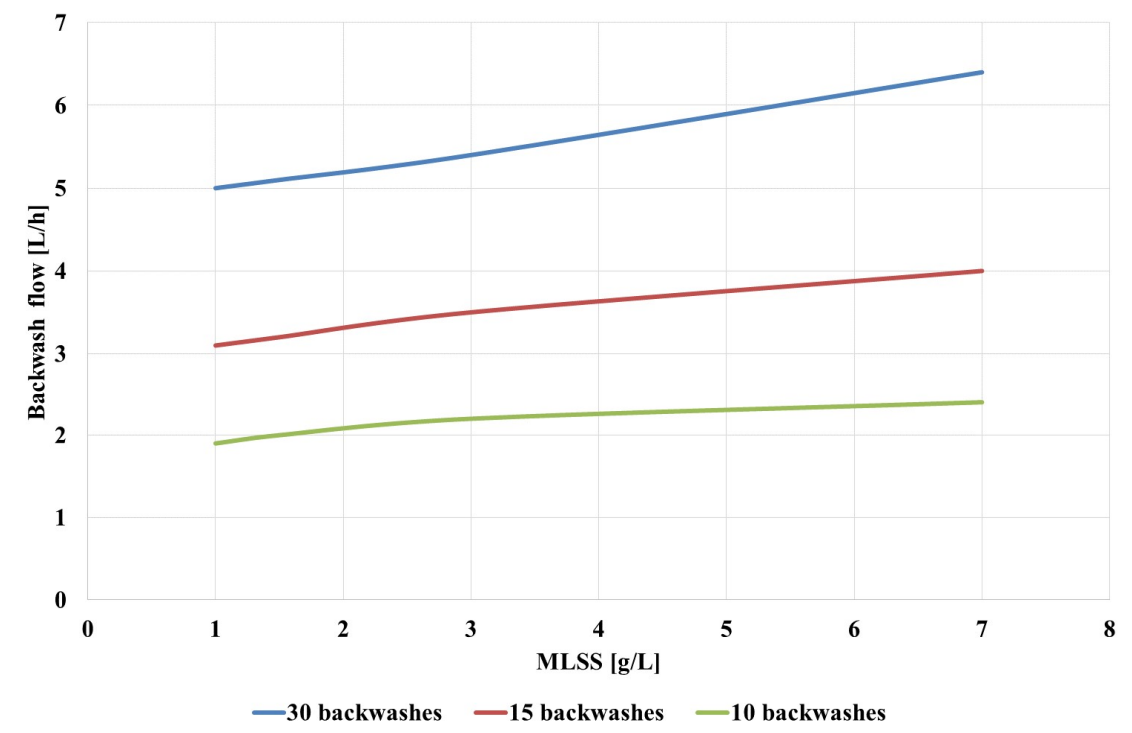

Fig.6. Backwash flow intensity in case of different backwash frequency 
Figure 6 demonstrates dependencies of backwashing flow (of various backwash frequencies) to suspended solids concentration in the feed water. The increase in MLSS tends to increase in water consumption due to increase of frequency of backwashes and washing time.

Figures 7-9 gives examples of evaluation of "useful" permeate flow related to MLSS during operation of the module for various backwash frequencies performed at $0.2 \mathrm{MPa}$. Figure 7 refers to frequency of 30 backwashes, figure 8 - to 15 backwashes, and figure 9 to 10 backwashes. There are four graphs at each of these figures presenting the following: a flow of single cycle of filtration $\left(\mathrm{Q}_{1 \mathrm{f}}\right)$; a flow used for backwash $\left(\mathrm{Q}_{\mathrm{w}}\right)$; a total flow $\left(\mathrm{Q}_{\mathrm{tot}}\right)$ that is a sum of $\mathrm{Q}_{1 \mathrm{f}}$ and $\mathrm{Q}_{\mathrm{w}}$; a useful flow $\left(\mathrm{Q}_{\mathrm{u}}\right)$, which is a difference between $\mathrm{Q}_{1 \mathrm{f}}$ and $\mathrm{Q}_{\mathrm{w}}$

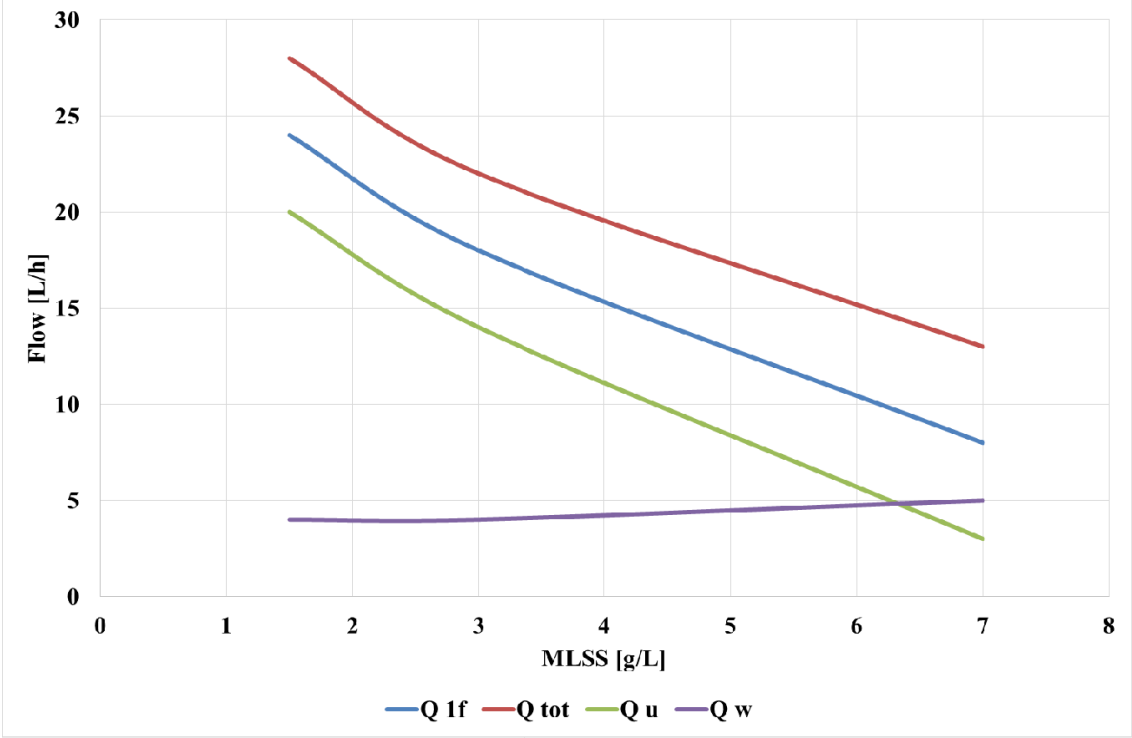

Fig. 7. Dependencies of flows to MLSS in condition of 30 backwashes

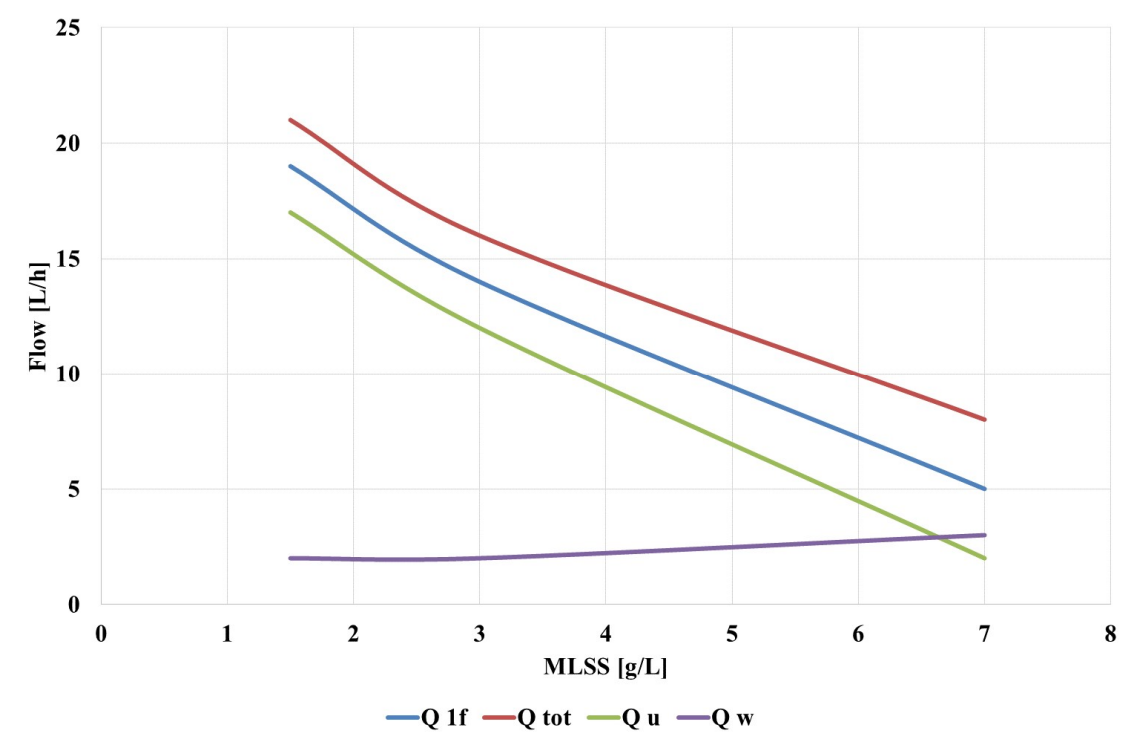

Fig. 8. Dependencies of flows to MLSS in condition of 15 backwashes 


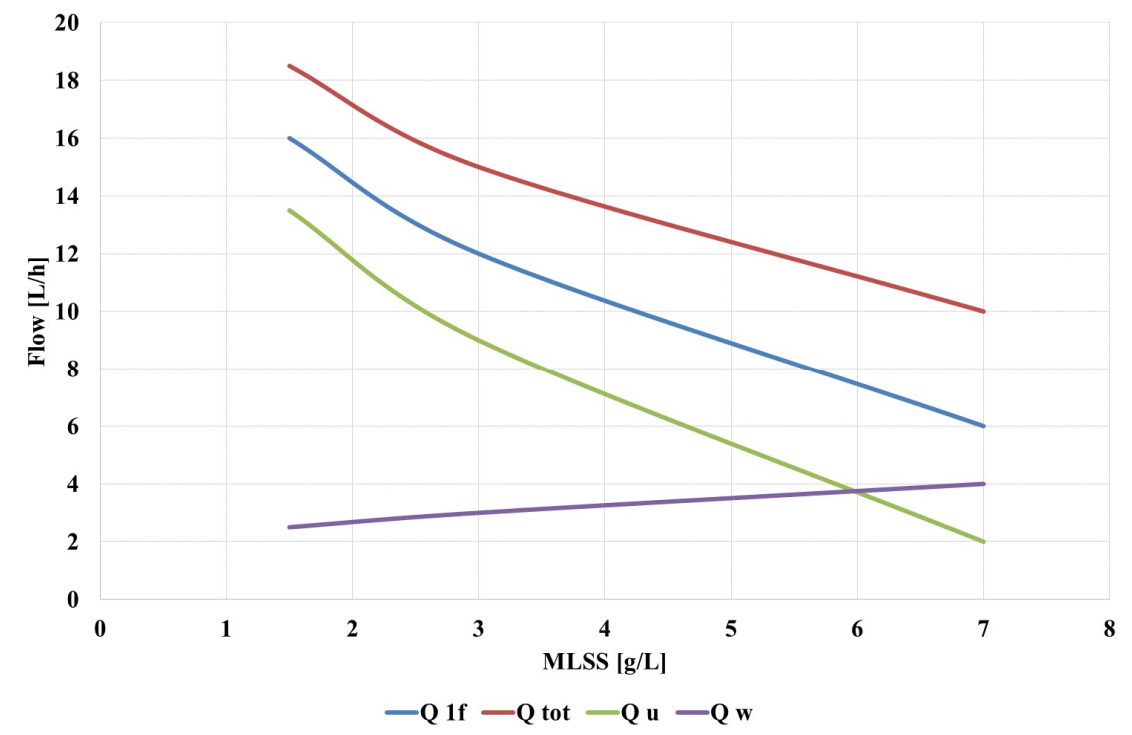

Fig. 9. Dependencies of flows to MLSS in condition of 10 backwashes

Having an optimum operation mode determined the next step of the research was to define the entire energy consumed and the specific energy consumption per flow. Within this we assumed that according to Q-H function of centrifugal pumps electric power required to pump up $1 \mathrm{~m}^{3} / \mathrm{h}$ at $1 \mathrm{~m}$ of height is $0.7 \mathrm{~kW}$. Figure 10 shows values of specific energy consumption calculated for the useful flow values shown on figures 7-9.

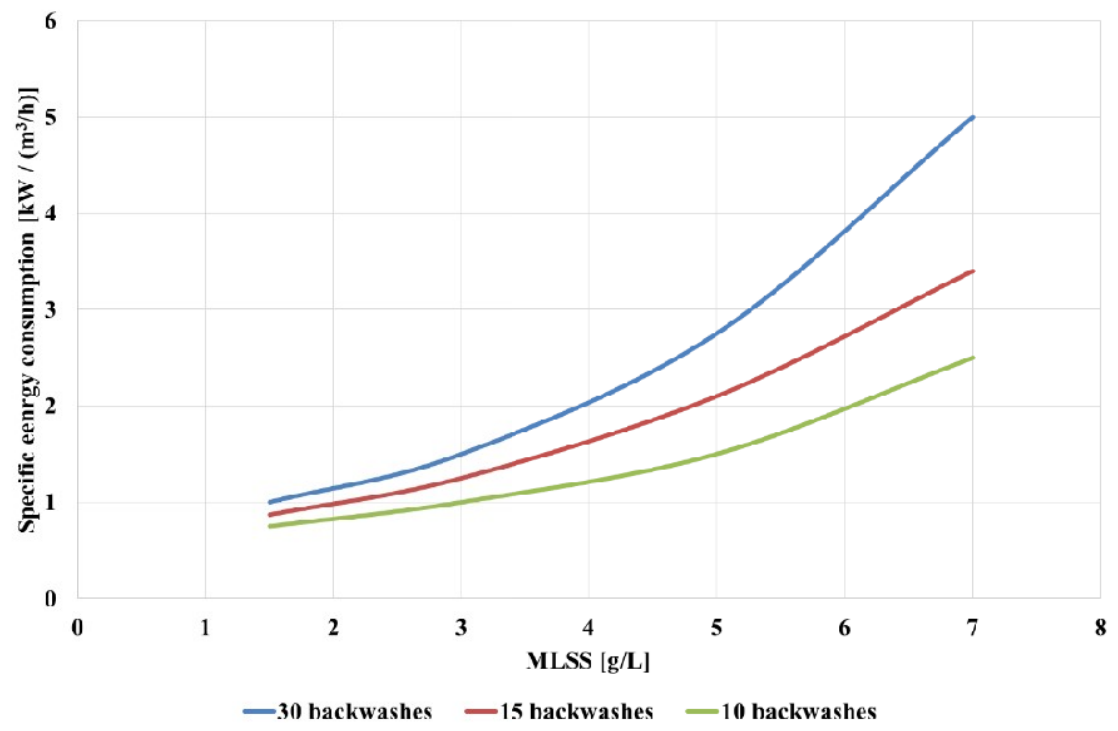

Fig.10. Specific energy consumption of the treatment process

Afterwards real energy consumption (figure 11) to provide useful flow was defined. That means that real energy consumption should sum up both power for filtration and power for backwash, so the specific energy consumption can be determined as the relation of real power consumption to useful flow. 


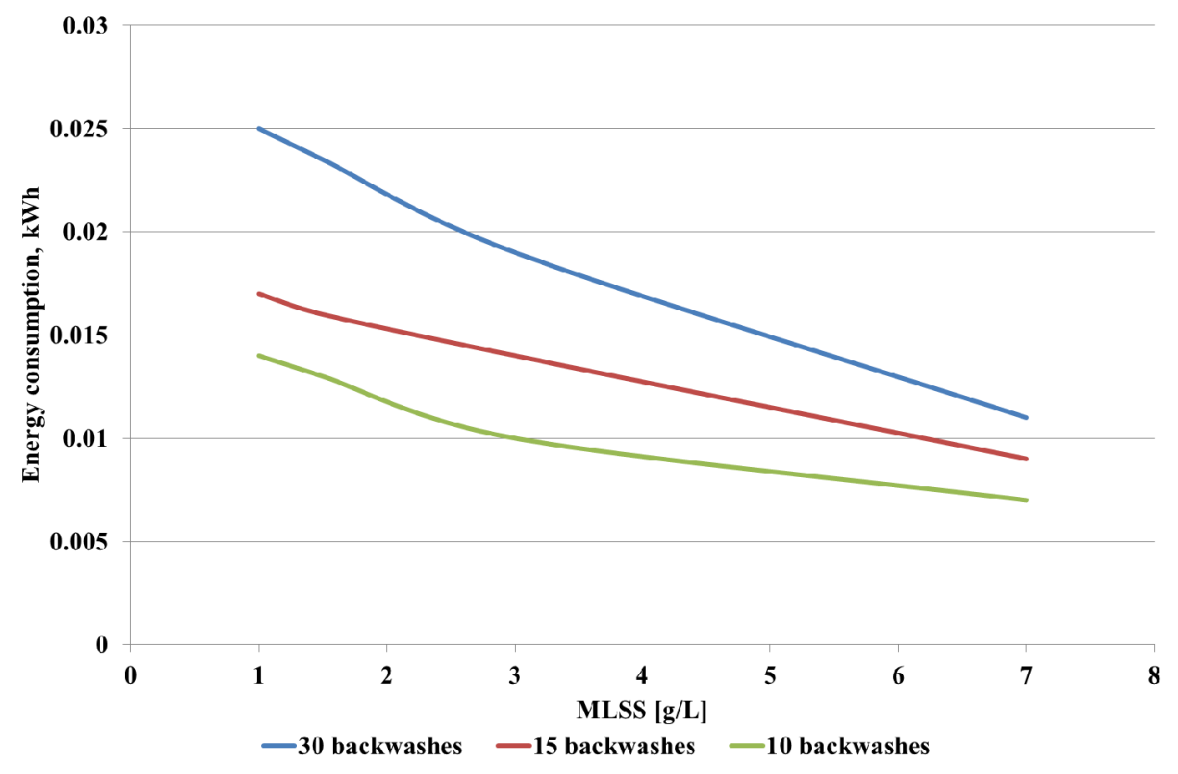

Fig. 11. Energy consumption.

It is important to note, that specific energy consumption at low MLSS value is more or less similar for different length of filtration cycle. Growth of MLSS provided a respective increase of specific energy consumption, however, the longer cycle of filtration (that means less amount of backwashing and less consumed water correspondingly) resulted in lower specific energy consumption with approx. 2 times difference if we compare 30 and 10 backwash mode respectively.

\section{Conclusions}

1. Permeate effluent has the most intensity within the first 3 minutes of operation, no matter what is the value of MLSS.

2. After 8 minutes of filtration, permeate production plummets due to accumulated fouling on membrane's surface (at high MLSS values in paeticular).

3. Shorter filtration cycles provides higher overall amount of filtered water, however it also means more frequent backwashes that increase water needs for that purpose.

4. Specific energy consumption has a strong relation to MLSS values and operation mode. Less frequent backwashing helps to reduce specific energy consumption by $10 \%$ at low MLSS values, and up to $50 \%$ at high MLSS values.

5. Further research is urgently needed to fit obtained results to various concentrations of pollutants in feed wastewater.

This work was financially supported by Ministry of Science and Higher Education of the Russian Federation (\#MK-519.2019.8). 


\section{References}

1. N. O. Yigit, G. Civelekoglu, I. Harman, H. Koseoglu, M. Kitis, Desalination, 237, 346356 (2009)

2. M. S. Nawaz, F. Parveen, S. J. Khan, N. P. Hankins, J. of Water Process Eng., 31, 100861 (2019)

3. Z. Cui, J. Wang, H. Zhang, H.H. Ngo, D. Kang, Biores. Tech., 269, 355-362 (2018)

4. K. Kimura, H. Uchida, Water Research, 1501, 21-28 (2019)

5. N. Makisha, A. Kulakov, MATEC Web of Conferences, 178, 09018 (2018)

6. G. Blandin, C. Gautier, M. S. Toran, H. Monclús, J. Comas, Chem. Eng. J., 3391, 268277 (2019)

7. I. Ruigómez, E. González, S. Guerra, L.E. Rodríguez-Gómez, L. Vera, J. of Membrane Sci., 52615, 181-190 (2017)

8. Z. Yin, V.V. Tarabara, I. Xagoraraki, Water Research, 881, $750-757$ (2016)

9. L. Vera, E. González, O. Díaz, S. Delgado, J. of Membrane Sci., 47015, 504-512 (2014)

10. Z. Cui, J. Wang, H. Zhang, H. Jia, J. of Membrane Sci., 53015, 132-145 (2017)

11. N. A. Makisha, Construction: science and education, 10(1), 6 (2020) 